\title{
Whole-tumor ADC histogram analysis for differentiating endometriosis- related tumors: seromucinous borderline tumor, clear cell carcinoma and endometrioid carcinoma
}

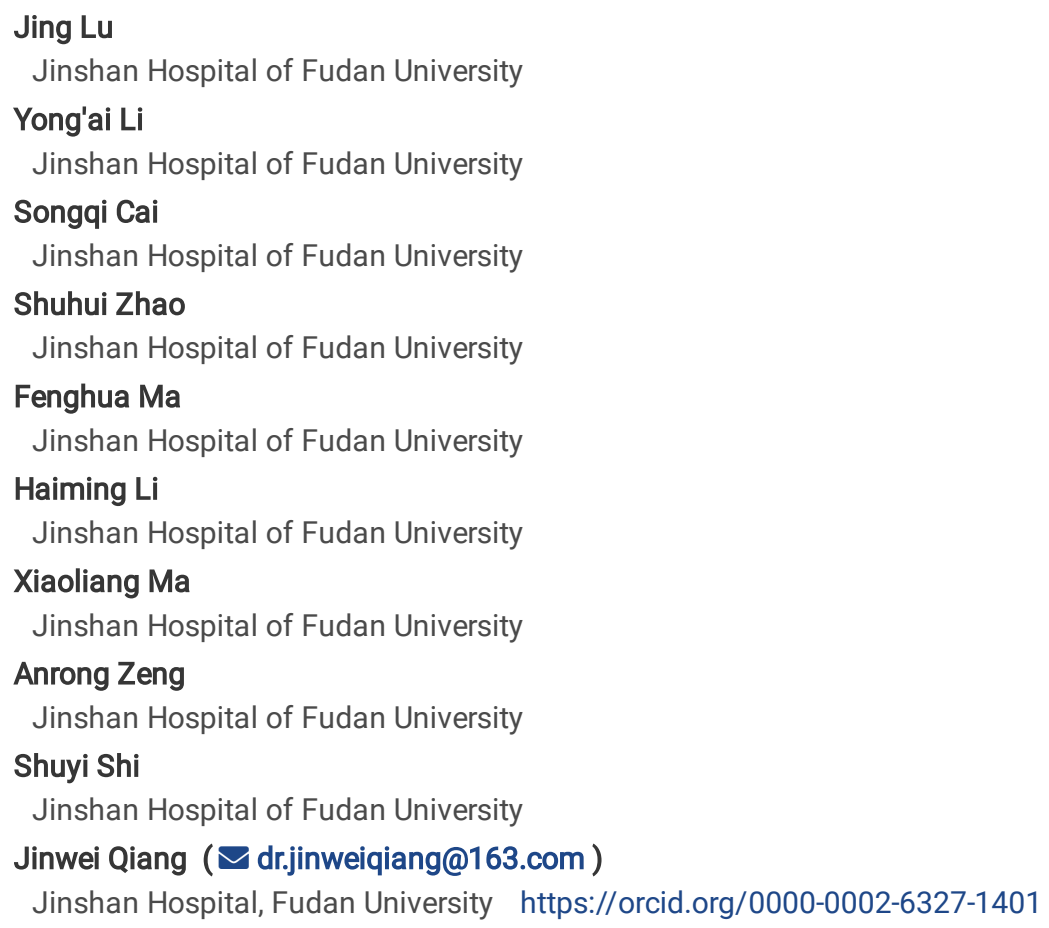

\section{Research}

Keywords: Seromucinous borderline tumor, Clear cell carcinoma, Endometrioid carcinoma, Magnetic resonance imaging, Apparent diffusion coefficient, Histogram analysis.

Posted Date: May 20th, 2021

DOI: https://doi.org/10.21203/rs.3.rs-539019/v1

License: (c) (i) This work is licensed under a Creative Commons Attribution 4.0 International License. Read Full License 


\section{Abstract}

Background: In view of the similar MRI findings of endometriosis-related ovarian neoplasms, this study aimed to investigate the feasibility of whole-tumor apparent diffusion coefficient (ADC) histogram analysis for differentiating endometriosis-related tumors: seromucinous borderline tumor (SMBT), clear cell carcinoma (CCC) and endometrioid carcinoma (EC).

Methods: A total of 85 patients (22 with SMBT, 42 with CCC and 21 with EC) were retrospectively enrolled. Clinical data including age, stage, laterality, solid component $\mathrm{ADC}\left(\mathrm{ADC}_{\mathrm{SC}}\right)$ and whole-tumor $\mathrm{ADC}$ histogram-derived parameters, such as the volume, $\mathrm{ADC}$ mean, the 10 th, 50 th and 90th percentile ADCs, inhomogeneity, skewness, kurtosis and entropy, were compared among SMBT, CCC and EC. The diagnostic efficacy of these parameters was evaluated using receiver operating characteristic curve analysis.

Results: There were significant differences in age, $A D C_{S C}$ and histogram parameters including volume, $A D C_{\text {mean }}$, the 10 th, 50 th and 90 th percentile ADCs among three kinds of tumors (all $p<0.05$ ). Patients with SMBT were significantly younger than patients with CCC/EC. The significantly higher $\mathrm{ADC}_{\mathrm{SC}}$ and smaller volume were found in $\mathrm{SMBT}$ than in $\mathrm{CCC} / \mathrm{EC}$. The $\mathrm{ADC}_{\text {mean }}$ was significantly higher in $\mathrm{CCC}$ than in $\mathrm{EC}$. The 10 th percentile ADC was significantly lower in EC than in SMBT/CCC. The 50th and 90th percentile ADCs were significantly higher in CCC than in SMBT/EC. For differentiating SMBT from CCC, AUCs of the $A_{D C}$, volume, the 50th and 90th percentile ADCs were 0.97, 0.86, 0.72 and 0.81, respectively. For differentiating SMBT from EC, AUCs of the $A D C_{S C}$, volume and the 10 th percentile $A D C$ were $0.97,0.71$ and 0.72 , respectively. For differentiating $C C C$ from $E C, A U C s$ of the $A D C_{\text {mean }}$, the 10 th, 50 th and 90 th percentile $A D C s$ were $0.79,0.72,0.81$ and 0.85 , respectively.

Conclusions: Similar with $A D C_{S C}$, whole-tumor $A D C$ histogram analysis was valuable for differentiating endometriosis-related tumors. It was helpful for the 50th, 90th percentile ADCs and volume in differentiating SMBT from CCC; for the volume and 10th percentile ADC in differentiating SMBT from EC, and for the $A D C_{\text {mean }}$, the 10th, 50th and 90th percentile ADCs in differentiating CCC from EC.

\section{Introduction}

Epithelial ovarian tumors are now divided into seven types according to the 2014 World Health Organization's classification of tumors of the female reproductive organs: serous, mucinous, seromucinous, endometrioid, clear cell and Brenner tumors, and undifferentiated carcinomas ${ }^{[1]}$. Seromucinous tumors of the ovary were formerly classified as the Mullerian or endocervical subtype of mucinous tumors ${ }^{[2]}$. Along with clear cell carcinoma (CCC) and endometrioid carcinoma (EC), seromucinous tumors are reported to be a type of endometriosis-related ovarian neoplasm [3]. Seromucinous borderline tumor (SMBT) is reported accounting for $7.6 \%$ of all borderline ovarian tumors ${ }^{[2,4]}$. Approximately $30-50 \%$ of SMBTs are associated with endometriosis ${ }^{[4,5]}$. Up to $90 \%$ of cases present as International Federation of Gynecology and Obstetrics (FIGO) stage I, and the prognosis is excellent even for advanced stage disease ${ }^{[5]}$. Patients who are initially treated with cystectomy, particularly those of reproductive age, can be managed by conservative observation if no residual lesions are identified on imaging studies ${ }^{[5]}$. However, CCC and EC are associated with a poorer prognosis. Furthermore, according to the National Comprehensive Cancer Network (NCCN) guidelines, the fertility preservation is not recommended for any stages of CCC, but is feasible for early EC patients ${ }^{[6]}$. Therefore, it is clinically important but often difficult to preoperatively differentiate SMBT from other endometriosis-related ovarian tumors such as CCC and EC ${ }^{[4]}$, and to correctly discriminating between CCC and $\mathrm{EC}^{[7]}$. Studies have shown that magnetic resonance imaging (MRI) findings in SMBT, CCC and EC often overlap, with appearing as a large cystic mass with intracystic vascularized mural nodules and/or solid components ${ }^{[4,8,9]}$. The cystic components are bloody, showing a hyperintensity on T1- and T2-weighted images (T1/T2WI) ${ }^{[10]}$. The mean apparent diffusion coefficient (ADC) value of the solid components was most useful parameter for differentiating SMBT from CCC and EC ${ }^{[4]}$. However, this traditional ADC was obtained through a circular region of interest (ROI) on the solid components without taking into account the heterogeneous distribution of the voxel-wise ADC within an entire tumor volume. In addition, few studies have been reported for preoperatively differentiating between CCC and EC.

Histogram analysis of the ADC can interrogate the biologic heterogeneity of tumors by classifying domains of different diffusion ${ }^{[11]}$. Recently, solid-tumor histogram analysis of the ADC has been used for differential diagnosis and prediction of chemotherapy response in epithelial ovarian tumors $^{[11-13]}$; whole-tumor histogram analysis of the ADC has also been studied in diagnostic, grading, prognostic and predictive applications in hepatocellular carcinoma, sinonasal malignant tumors, malignant parotid gland tumors, glioblastoma multiforme and ovarian cancers ${ }^{[14-18]}$.

In view of the similar MRI findings of the three types of endometriosis-related ovarian neoplasms, this study aimed to investigate the feasibility of whole-tumor histogram analysis of the ADC in differentiation of SMBT, CCC and EC by comparing with the traditional ADC of solid components $\left(A D C_{S C}\right)$.

\section{Material And Methods}

\section{Study Population}


This retrospective study was approved by the institutional review board, and informed consent was waived for all patients. Between February 2011 and February 2019, patients with SMBT, CCC and EC proven by histology were reviewed. The inclusion criteria were as follows: 1) patients undergoing abdominopelvic MRI scanning before treatment; 2) patients' MRI including diffusion weighted imaging (DWI) with $b$ factors of 0,1000 $\mathrm{s} / \mathrm{mm}^{2} ; 3$ ) tumors were removed by surgery and proven endometriosis-related by histopathology. The exclusion criteria were as follows: 1 ) tumors without histopathologically confirmed endometriosis; 2) patients with a tumor too small to be detected on MRI; and 3) MRI scans with poor image quality. Patients' clinical data, including age, laterality, and FIGO stage, were recorded.

\section{MRI Technique}

MRI was performed with a 1.5-T scanner (Avanto, Siemens, Erlangen, Germany) with a phased array coil. The patients lay in the supine position and breathed freely during the acquisition. The scanning range was from the inferior pubic symphysis to the renal hilum and extended beyond the dome of the tumor in cases of large masses. The scanning sequences and parameters are listed in Table 1. Axial DWI was performed using single-shot echo planar imaging sequence with the following diffusion gradient b factors: 0 and $1000 \mathrm{~s} / \mathrm{mm}^{2}$. Axial and sagittal contrastenhanced imaging were scanned after administering gadolinium chelate (Gd-DTPA, Magnevist; Bayer Schering, Berlin, Germany) at a dose of 0.2 $\mathrm{mL} / \mathrm{kg}$ body weight.

Table1. MRI sequences and parameters in patients with SMBT, CCC and EC

\begin{tabular}{lllll}
\hline Parameters & T1WI & T2WI & T1WI-FS & DWI \\
\hline Repetition time (ms) & 340 & 8000 & 196 & 3200 \\
Echo time (ms) & 10 & 83 & 2.9 & 87 \\
Section thickness (mm) & 5 & 5 & 5 & 5 \\
Intersection gap (mm) & 1.5 & 1.5 & 1.5 & 1.5 \\
Field of view (mm) & $238 \times 280$ & $238 \times 280$ & $238 \times 280$ & $238 \times 280$ \\
Matrix & $256 \times 256$ & $256 \times 256$ & $256 \times 256$ & $128 \times 128$ \\
Acquisition time (s) & 120 & 175 & 40 & 166 \\
$b$ factors (s/mm ${ }^{2}$ ) & - & - & - & 0,1000 \\
\hline
\end{tabular}

SMBT, seromucinous borderline tumor; CCC, clear cell carcinoma; EC, endometrioid carcinoma; MRI, magnetic resonance imaging; T1WI, T1-weighted imaging; T2WI, T2-weighted imaging; FS, fat saturation; DWI, diffusion-weighted imaging.

\section{Imaging Analysis}

MR images were independently analyzed by two radiologists (radiologist 1 and 2, with 6 and 11 years of experience in gynecological imaging, respectively) who were blinded to the histopathology. Images were analyzed again by radiologist 1 after an interval of 1 month. The $A D C_{S C}$ value was measured on the slice with largest solid component of tumor of the ADC map, and an elliptical ROI was drawn as large as possible at the solid component of the tumor, carefully avoiding areas of hemorrhage and necrosis, and major vascular structures. Whole-tumor histogram analysis was performed on the ADC map using Fire Voxel (CAI2R, New York University, NY, USA) according to the methods described in a previous study ${ }^{[11]}$. The ROI was manually delineated slice-by-slice along the contour of the tumor, referring to T2WI (Fig 1). Then, the volume of interest of the ADC was postprocessed automatically for the histogram analysis. The ADC value for each pixel was recorded. An ADC histogram was generated in which the contribution of each individual tumor was weighted according to its volume, with a bin width of $1 \times 10^{-5} \mathrm{~mm}^{2} / \mathrm{s}$. Histogram-derived parameters, such as volume, $A D C_{\text {mean }}$, the 10 th, 50th and 90 th percentiles, skewness, kurtosis, inhomogeneity and entropy were calculated using SPSS software (version 23.0, SPSS, Inc., Chicago, IL).

\section{Statistical Analysis}

Statistical analyses were performed with SPSS software. The normality of variables was tested using Shapiro-Wilk test. Continuous variables with a normal distribution were presented as the mean \pm standard deviation, while non-normally distributed variables were reported as the median (interquartile range). The intra- and interclass correlation coefficients (ICCs) were calculated to evaluate intra- and interobserver agreements, 
respectively (ICC: 0.00-0.20, poor correlation; 0.21-0.40, fair; 0.41-0.60, moderate; 0.61-0.80, good; and 0.81-1.00, excellent). Categorical variables, including laterality and FIGO stage, were compared using the chi-square test. The One-way ANOVA or Kruskal-Wallis was performed to compare the differences in age, $\mathrm{ADC}_{\mathrm{SC}}$ and histogram-derived parameters. Receiver operating characteristic (ROC) curve analysis (MedCalc Software, Mariakerke, Belgium) was used to assess the diagnostic performance and determine optimal cutoff values of the histogram-derived parameters for differentiating SMBT from CCC or EC, and differentiating CCC from EC. The Delong test was used to compare differences in the area under the curve (AUC) for each parameter. A $p$ value less than 0.05 was regarded as statistically significant.

\section{Results}

\section{Patient Demographics}

A total of 85 patients with 22 SMBTs, 42 CCCs and 21 ECs were enrolled. Clinical features of patients with SMBT and CCC/EC are summarized in Table 2. There were significant differences of ages among three groups of patients $(p<0.001)$. Thereinto, patients with SMBT were significantly younger (39.91 \pm 12.08 years) than patients with CCC (51.48 \pm 9.52 years) and EC $(52.24 \pm 8.15$ years) (both $p<0.001)$. There was no significant difference between patients with CCC and EC $(p=1.000)$. Most patients with SMBT, CCC and EC were at early stage (stage I and II) and all the patients had a unilateral tumor. No significant differences were observed in the FIGO stage and laterality among three groups $(p=0.312,0.722$, respectively).

Table2. Clinical features of patients with SMBT, CCC and EC

\begin{tabular}{lllll}
\hline Features & SMBT $(\mathrm{n}=22)$ & CCC $(\mathrm{n}=42)$ & EC $(\mathrm{n}=21)$ & $p$ value \\
\hline Age (mean, y) & $39.91 \pm 12.08^{\$ £}$ & $51.48 \pm 9.52^{\$}$ & $52.24 \pm 8.15^{\ddagger}$ & $<0.001^{*}$ \\
Laterality & & & & $0.722^{\#}$ \\
Right & 14 & 23 & 11 & \\
Left & 8 & 19 & 10 & $0.312^{\S}$ \\
FIGO stage & & & & \\
I & 18 & 41 & 16 & \\
II & 2 & 0 & 5 & \\
III & 2 & 1 & 0 & \\
IV & 0 & 0 & 0 & \\
\hline
\end{tabular}

FIGO, International Federation of Gynecology and Obstetrics. * One-way ANOVA; \# Chi-square test; § Fisher's precision probability test (FIGO I+II vs III+IV); \$, £ There was significant difference between the two groups.

\section{Solid component $\mathrm{ADC}\left(\mathrm{ADC} \mathrm{SC}_{\mathrm{S}}\right)$ and whole-tumor $\mathrm{ADC}$ histogram parameters}

The intra- and interobserver agreement was excellent for the $\mathrm{ADC}_{\mathrm{SC}}$ and histogram parameters, with ICCs of 0.961 (95\% confidence interval (CI), $0.941,0.974)$ and $0.980(95 \% \mathrm{Cl}, 0.970,0.987)$, respectively. The results of the first measurement by radiologist 1 were used for further analyses.

There were significant differences in the $\mathrm{ADC}_{\mathrm{SC}}$ and whole-tumor histogram parameters including the volume, $A D C_{\mathrm{mean}}$, the 10 th, 50 th and 90 th percentile ADCs among three kinds of tumors (all $p<0.01$ ) (Table 3). The $\mathrm{ADC}_{\mathrm{SC}}$ was significantly higher in SMBT than in CCC and EC (both $p<$ $0.001)$, but was no significant difference between CCC and EC $(p=0.328)$. The volume was significantly smaller in SMBT than in CCC ( $p<0.001)$ and EC ( $p=0.024)$, but was no significant difference between $\mathrm{CCC}$ and $\mathrm{EC}(p=0.559)$. The $\mathrm{ADC}_{\text {mean }}$ was significantly higher in $\mathrm{CCC}$ than in $\mathrm{EC}(p$ $<0.001$ ), but were no significant differences between SMBT and CCC/EC ( $p=0.050$ and 0.418, respectively). The 10th percentile ADC was significantly lower in EC than in SMBT $(p=0.033)$ and CCC $(p=0.011)$, but was no significant difference between SMBT and CCC $(p=1.000)$. The 50th and 90th percentile ADCs were significantly higher in CCC than in SMBT and EC ( $p=0.015, p<0.001$ and both $p<0.001$, respectively), but were no significant differences between SMBT and EC ( $p=0.814$ and 1.000 , respectively). There were no significant differences found in other histogram-derived parameters including the skewness, kurtosis, inhomogeneity or entropy among three tumors. 
Table3. ADC of solid components and whole-tumor ADC histogram parameters in SMBT, CCC and EC

\begin{tabular}{|c|c|c|c|c|}
\hline trameters & $\operatorname{SMBT}(\mathrm{n}=22)$ & $\operatorname{CCC}(n=42)$ & $\mathrm{EC}(\mathrm{n}=21)$ & $p$ value \\
\hline DCsc* & $1.8(1.7,1.9)^{\$ €}$ & $1.2(1.0,1.4)^{\$}$ & $1.0(1.0,1.2)^{£}$ & $<0.001$ \\
\hline Volume $\left(\mathrm{cm}^{3}\right)$ & $187.6(76.0,249.9)^{\$ €}$ & $537.0(338.9,822.0)^{\$}$ & $280.1(140.3,1022.2)^{£}$ & $<0.001$ \\
\hline L0th percentile ADC* & $1.8(1.5,2.0)^{£}$ & $1.7(1.3,2.2)^{\&}$ & $1.2(1.0,1.5)^{£ \&}$ & 0.008 \\
\hline ¡0th percentile ADC* & $2.1(1.7,2.3)^{\$}$ & $2.5(2.2,2.6)^{\$ \&}$ & $1.9(1.6,2.2)^{\S}$ & $<0.001$ \\
\hline Kurtosis & $3.1(2.4,6.2)$ & $2.6(-0.5,7.6)$ & $1.4(-0.9,4.8)$ & 0.104 \\
\hline Inhomogeneity & $0.1(0.1,0.1)$ & $0.2(0.1,0.2)$ & $0.1(0.1,0.2)$ & 0.052 \\
\hline Entropy & $3.5 \pm 0.4$ & $3.6 \pm 0.5$ & $3.8 \pm 0.5$ & 0.115 \\
\hline
\end{tabular}

ADC, apparent diffusion coefficient; ADCsc, solid component ADC; $p$ value, One-way ANOVA or Kruskal-Wallis. $* \times 10^{-3} \mathrm{~mm}^{2} / \mathrm{s} ;{ }^{\$}, £, \&$ There was significant difference between the two groups.

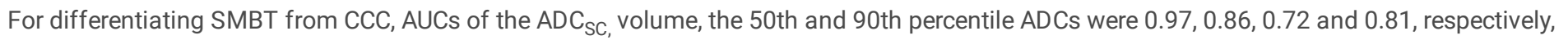
with a significant difference between $\mathrm{ADC}_{\mathrm{SC}}$ and the 50th percentile $\mathrm{ADC}(p=0.003)$ (Table 4, Fig 2); and no significant differences between $\mathrm{ADC}_{\mathrm{SC}}$ and volume, or 90 th percentile $\mathrm{ADC}\left(p=0.097\right.$ and 0.053 , respectively). For differentiating SMBT from $E C$, the $A D C_{S C}$ had a significantly larger AUC than the volume and 10th percentile $\mathrm{ADC}$ ( 0.97 vs 0.71 and $0.72, p=0.003$ and 0.015 , respectively). For differentiating CCC from EC, the $A D C_{\text {mean }}$, the 10th, 50th and 90th percentile ADCs had AUCs of $0.79,0.72,0.81$ and 0.85 , without significant AUC differences among the four histogram-derived parameters (all $p>0.05$ ).

Table 4. Diagnostic performance of ADC of solid components and whole-tumor histogram parameters in differentiating SMBT, CCC and EC 


\begin{tabular}{|c|c|c|c|c|c|c|c|c|c|c|c|c|}
\hline \multirow[t]{2}{*}{ Parameters } & \multicolumn{4}{|c|}{ SMBT vs CCC } & \multicolumn{4}{|c|}{ SMBT vs EC } & \multicolumn{4}{|c|}{ CCC vs EC } \\
\hline & AUC & $\begin{array}{l}\text { Sensitivity } \\
\text { (\%) }\end{array}$ & $\begin{array}{l}\text { Specificity } \\
(\%)\end{array}$ & $\begin{array}{l}\text { Cutoff } \\
\text { value }\end{array}$ & AUC & $\begin{array}{l}\text { Sensitivity } \\
\text { (\%) }\end{array}$ & $\begin{array}{l}\text { Specificity } \\
\text { (\%) }\end{array}$ & $\begin{array}{l}\text { Cutoff } \\
\text { value }\end{array}$ & AUC & $\begin{array}{l}\text { Sensitivity } \\
\text { (\%) }\end{array}$ & $\begin{array}{l}\text { Specificity } \\
\text { (\%) }\end{array}$ & $\begin{array}{l}\text { Cutoff } \\
\text { value }\end{array}$ \\
\hline ADCsc* & $0.97^{\$}$ & 94.1 & 94.9 & 1.5 & $\begin{array}{l}0.97^{\$} \\
€\end{array}$ & 94.1 & 94.4 & 1.5 & - & - & - & - \\
\hline $\begin{array}{l}\text { Volume } \\
\left(\mathrm{cm}^{3}\right)\end{array}$ & 0.86 & 77.3 & 88.1 & 237.1 & $0.71^{\$}$ & 77.3 & 66.7 & 237.1 & - & - & - & - \\
\hline ADCmean* & - & - & - & - & - & - & - & - & 0.79 & 76.2 & 76.2 & 2.0 \\
\hline $\begin{array}{l}\text { 10th } \\
\text { percentile }\end{array}$ & - & - & - & - & $0.72^{£}$ & 77.3 & 76.2 & 1.5 & 0.72 & 85.7 & 52.4 & 1.2 \\
\hline ADC* & & & & & & & & & & & & \\
\hline $\begin{array}{l}\text { 50th } \\
\text { percentile } \\
\text { ADC* }\end{array}$ & $0.72^{\$}$ & 77.3 & 69.1 & 2.2 & - & - & - & - & 0.81 & 61.9 & 95.2 & 2.4 \\
\hline $\begin{array}{l}\text { 90th } \\
\text { percentile } \\
\text { ADC* }\end{array}$ & 0.81 & 68.2 & 88.1 & 2.5 & - & - & - & - & 0.85 & 73.8 & 90.5 & 2.6 \\
\hline
\end{tabular}

AUC, area under the curve.

$*, \times 10^{-3} \mathrm{~mm}^{2} / \mathrm{s} ; \$,{ }^{\ddagger}$ There was significant difference in AUC between the two parameters.

\section{Discussion}

Although traditional ADC showed perfect diagnostic performance for differentiating between SMBT and CCC/EC, the present study demonstrated the potential of whole-tumor ADC histogram analysis to differentiate CCC from EC.

There were no significant differences between CCC and EC in the clinical characteristics, such as age, laterality of tumor and FIGO stages, which was similar with previous studies ${ }^{[7,19]}$. Although it has been reported that SMBTs can involve the bilateral ovaries in up to $40 \%$ of patients ${ }^{[5]}$, in our study, all SMBTs happened to occur in a unilateral ovary. The early-stage tumors and small sample size might explain the inconsistency. The mean age at diagnosis was significantly younger in patients with SMBT (39.91 \pm 12.08 years) than in patients with CCC/EC (51.48 $\pm 9.52,52.24 \pm$ 8.15 years). This finding was consistent with a previous review that reported an average age of $34-44$ years for patients with SMBT ${ }^{[2]}$. The volume of SMBT $\left(187.6 \mathrm{~cm}^{3}\right)$ in our study was significantly smaller than that of CCC/EC $\left(537.0 \mathrm{~cm}^{3}, 280.1 \mathrm{~cm}^{3}\right)$. This finding was similar to those of previous studies that found a significantly smaller size of tumor and its solid components in SMBT than in other endometriosis-related malignant tumors $^{[2,4]}$.

A previous study showed that SMBT had a higher $\mathrm{ADC}_{\mathrm{SC}}$ value than $\mathrm{CCC}$ and $\mathrm{EC}^{[4]}$. This is generally understood as a consequence of a greater tumor cellularity in malignancies that limits the free diffusion of water molecules ${ }^{[20]}$. Our study also confirmed that the $\mathrm{ADC}_{\mathrm{SC}} \mathrm{value}$ was significantly higher in SMBT than in CCC and EC and yielded a perfect performance for differentiating SMBT from CCC and EC with both AUCs of 0.97. However, MRI findings of CCC and EC often overlap and few studies succeeded in differentiating them with a moderate diagnostic performance ${ }^{[7,19,21]}$. Morphological features of EC, such as multilocular cysts, broad-based (smaller height/width ratio), multifocal or continuing growth pattern of the mural nodules and presence of ascites, were reported in different studies with the AUCs from 0.50 to $0.76^{[7,19]}$. Ono reported that the mean $\mathrm{ADC}_{\mathrm{SC}}$ value was $1.24 \pm 0.17 \times 10^{-3} \mathrm{~mm}^{2} / \mathrm{s}$ in 17 cases of $\mathrm{CCC}$, which was significantly higher than that $\left(0.84 \pm 0.10 \times 10^{-3} \mathrm{~mm}^{2} / \mathrm{s}\right)$ of 14 cases of EC ${ }^{[22]}$. In our study, the $\mathrm{ADC}_{\mathrm{SC}}$ value was $1.2 \times 10^{-3} \mathrm{~mm}^{2} / \mathrm{s}$ in 42 cases of CCC, comparing with $1.0 \times 10^{-3} \mathrm{~mm}^{2} / \mathrm{s}$ in $21 \mathrm{cases}$ of $\mathrm{EC}$ 
without significant difference. Larger sample sizes and tumors with histopathologically confirmed endometriosis might explain the difference between the two studies.

Although our whole-tumor histogram analysis showed that there were no significant $A D C_{\text {mean }}$ differences between the $S M B T$ and $C C C / E C$ groups,

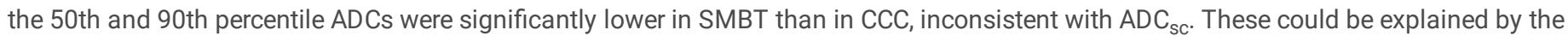
different ROI methods used. In addition to the solid components of tumors, cystic contents were also included in the whole-tumor histogram analysis that could compromise $\mathrm{ADC}_{\text {mean }}$ difference between $\mathrm{SMBT}$ and $\mathrm{CCC} / \mathrm{EC}$. The whole-tumor $\mathrm{ADC}$ histogram analysis can provide more detailed information on the diffusion characteristics of tumors than traditional ADC values ${ }^{[22]}$, allowing the evaluation of intratumoral heterogeneity ${ }^{[23]}$. Even though no significant differences were found in inhomogeneity, entropy, skewness or kurtosis among SMBT, CCC and EC, the $A D C_{\text {mean }}$, 10th, 50th and 90th percentile ADCs could be usful for differentiating CCC from EC and the 90th percentile ADC showed a largest AUC of 0.85. Previous studies suggested that the lower percentile ADCs might represent the hypercellular component of the tumor, while the higher percentile ADCs represent cystic content of tumor ${ }^{[14,24,25]}$. Our study showed a significantly lower 10 th percentile ADC in EC than in SMBT and CCC, indicating a greater tumor cellularity in EC, consistent with previous studies ${ }^{[4,20,21]}$; and a significantly higher 50 th and 90 th percentile $A D C s$ in CCC than in SMBT and EC, indicating the more cystic contents, and smaller overall and solid portion sizes in SMBT and EC ${ }^{[4]}$. Furthermore, previous studies showed that the ADC value of cystic content decreased as blood and protein concentrations increased ${ }^{[25]}$ and the ${ }^{\text {T1WI }}$ signal intensity of cystic contents was significantly higher in SMBT than in $\mathrm{CCC}^{[4]}$. These findings could be ascribe to hemorrhagic, mucinous and mucopurulent fluid in SMBT ${ }^{[5]}$, nonhemorrhagic serous fluid produced by the more malignant $\mathrm{CCC}^{[26]}$ and different transformation pathways and time phases between CCC and EC $[19,27,28]$.

Our study had several limitations. First, it was a retrospective study with a small sample size, especially in the sample of patients with SMBT. However, considering the rarity of SMBT, it might be difficult to perform a large cohort study. Second, some clinical information such as CA 125 was deficient in some cases as we enrolled patients for a long period of time. A history of menstruation and related serum tests may provide additional indications for diagnosis of endometriosis-related diseases. Third, only ADC histogram analyses were performed in our study. Seeing that endometriosis-related tumors undergo repeated cycles of hemorrhage, the varying age of the hemorrhage in the cysts tends to have a varied but characteristic signal on T1WI and T2WI. Histogram analysis of T1WI and T2WI might contribute to the differentiation of SMBT, CCC and EC.

\section{Conclusions}

In summary, although traditional $\mathrm{ADC}_{\mathrm{SC}}$ showed the perfect diagnostic performance in differentiating SMBT from CCC/EC, this study further demonstrated that whole-tumor ADC histogram analysis was able to differentiate endometriosis-related tumors, with volume for differentiating SMBT from CCC/EC and 90th percentile ADC for differentiating CCC from EC.

\section{List Of Abbreviations}

ADC: Apparent diffusion coefficient; ADCSC: ADC of solid components; AUC: Area under the curve; CCC: Clear cell carcinoma; DWI: Diffusion weighted imaging; EC: Endometrioid carcinoma; FIGO: International Federation of Gynecology and Obstetrics; ICC: Interclass correlation coefficient; MRI: Magnetic resonance imaging; ROI: Region of interest; ROC: Receiver operating characteristic; SMBT: Seromucinous borderline tumor.

\section{Declarations}

\section{Ethics approval and consent to participate}

This retrospective study was approved by the institutional review board of Jinshan Hospital, Fudan University, China (Approval number, 2018-30). The informed consent requirement was waived.

\section{Consent for publication}

Not applicable.

\section{Availability of data and materials}

We would not share the data and material used in this manuscript, because we need them for further research.

\section{Competing interests}

The authors declare that they have no competing interests. 


\section{Funding}

This work was supported by the National Natural Science Foundation of P.R. China [No. 81971579], Shanghai Municipal Commission of Science and Technology [No. 19411972000], Shanghai Municipal Health Commission [No. ZK2019B01] and Shanghai Jinshan District Health Commission [No.JSYQ201901].

\section{Authors' contributions}

$\mathrm{JL}$ and YAL conceived of the study, and participated in its design and drafted the manuscript. SQC, SHZ, FHM, HML and XLM participated in the data acquisition. ARZ and SYS participated in the data analysis. JWQ guaranteed the integrity of entire study. All authors read and approved the final manuscript.

\section{Acknowledgements}

Not applicable.

\section{Authors' information}

\begin{tabular}{ll} 
Jing Lu, & E-mail: wohelujing@sohu.com \\
\hline Yong'ai Li, & E-mail: pingyaoaiai@163.com \\
\hline Songqi Cai, & E-mail: csqbee@163.com \\
\hline Shuhui Zhao, & E-mail: zhaoshuhui1029@163.com \\
\hline Fenghua Ma, & E-mail: mafenghua9602@163.com \\
\hline Haiming Li, & E-mail: lihaiming0109@163.com \\
\hline Xiaoliang Ma, & E-mail: 810480378@qq.com \\
\hline Anrong Zeng, & E-mail: doctoranrongzeng@163.com \\
\hline Shuyi Shi, & E-mail: 775468231@qq.com
\end{tabular}

\section{References}

1. Kurman RJ. International agency for research on cancer, World Health Organization. WHO Classification of Tumours of Female Reproductive Organs. 4th ed. Lyon, France: International Agency for Research on Cancer; 2014.

2. Karpathiou G, Chauleur C, Corsini T, et al. Seromucinous ovarian tumor a comparison with the rest of ovarian epithelial tumors. Annals of Diagnostic Pathology. 2017, 27: 28-33.

3. Maeda D, Shih I. Pathogenesis and the role of ARID1A mutation in endometriosis-related ovarian neoplasms. Adv Anat Pathol. 2013, 20: 4552.

4. Kurata Y, Kido A, Moribata Y, et al. Diagnostic performance of MR imaging findings and quantitative values in the differentiation of seromucinous borderline tumour from endometriosis-related malignant ovarian tumour. Eur Radiol. 2017, 27: 1695-1703.

5. Nagamine M, Mikami Y. Ovarian seromucinous tumors: pathogenesis, morphologic spectrum, and clinical i Diagnostics (Basel). $2020,10: 77$.

6. Armstrong D K, Alvarez R D, Bakkum-Gamez J N, et al. NCCN guidelines insights: ovarian cancer, Version 1.2019. J Natl Compr Canc Netw. 2019, 17: 896-909.

7. Li M, Tan J, Zhang Y, et al. Assessing CT imaging features combined with CEA and CA125 levels to identify endometriosis-associated ovarian cancer. Abdom Radiol (NY). 2020.

8. Han J W, Kim K A, Chang H Y, et al. Newly categorized seromucinous tumor of the ovary: magnetic resonance imaging $\mathrm{f} J \mathrm{~J}$ Comput Assist Tomogr. 2019, 43: 119-127.

9. Kurata Y, Kido A, Moribata Y, et al. Differentiation of seromucinous borderline tumor from serous borderline tumor on MR i Magn Reson Med Sci. 2018, 17: 211-217.

10. Liu T, Sumida D, Wada T, et al. A diagnostic challenge of seromucinous borderline tumor: a case report. Medicine (Baltimore). 2019, 98:

11. Lu J, Li H M, Cai S Q, et al. Prediction of platinum-based chemotherapy response in advanced high-grade serous ovarian cancer: ADC histogram analysis of primary t Acad Radiol. 2020: S1076-6332(20)30052-0.

12. He M, Song Y, Li H, et al. Histogram analysis comparison of monoexponential, advanced diffusion-weighted imaging, and dynamic contrastenhanced MRI for differentiating borderline from malignant epithelial ovarian t J Magn Reson Imaging. 2020. Online ahead of print. 
13. Li H M, Zhang R, Gu W Y, et al. Whole solid tumour volume histogram analysis of the apparent diffusion coefficient for differentiating highgrade from low-grade serous ovarian carcinoma: correlation with Ki-67 proliferation status. Clin Radiol. 2019, 74: 918-925.

14. Xu Y S, Liu H F, Xi D L, et al. Whole-lesion histogram analysis metrics of the apparent diffusion coefficient: a correlation study with histological grade of hepatocellular carcinoma. Abdom Radiol (NY). 2019, 44: 3089-3098.

15. Xiao Z, Tang Z, Zhang J, et al. Whole-tumor histogram analysis of monoexponential and advanced diffusion-weighted imaging for sinonasal malignant tumors: Correlations with histopathologic features. J Magn Reson Imaging. 2020, 51: 273-285.

16. Zhang Z, Song C, Zhang Y, et al. Apparent diffusion coefficient (ADC) histogram analysis: differentiation of benign from malignant parotid gland tumors using readout-segmented diffusion-weighted imaging. Dentomaxillofac Radiol. 2019, 48:

17. Pope W B, Kim H J, Huo J, et al. Recurrent glioblastoma multiforme: ADC histogram analysis predicts response to bevacizumab treatment. Radiology. 2009, 252: 182-189.

18. Kyriazi S, Collins D J, Messiou C, et al. Metastatic ovarian and primary peritoneal cancer: assessing chemotherapy response with diffusionweighted MR imaging-value of histogram analysis of apparent diffusion coefficients. Radiology. 2011, 261: $182-192$.

19. Morioka S, Kawaguchi R, Yamada Y, et al. Magnetic resonance imaging findings for discriminating clear cell carcinoma and endometrioid carcinoma of the ovary. J Ovarian Res. 2019, 12: 20.

20. Zhang Y D, Wu C J, Wang Q, et al. Comparison of utility of histogram apparent diffusion coefficient and R2* for differentiation of low-grade from high-grade clear cell renal cell c AJR Am J Roentgenol. 2015, 205: W193-W201.

21. Yumiko O T, Satoshi O, Takako Y, et al. MRI of endometriotic cysts in association with ovarian carcinoma. AJR Am J Roentgenol. 2010, 194: 355-361.

22. Ono T, Kishimoto K, Tajima S, et al. Apparent diffusion coefficient (ADC) values of serous, endometrioid, and clear cell carcinoma of the ovary: pathological correlation. Acta Radiol. 2020, 61: 992-1000.

23. Schob S, Meyer H, Dieckow J, et al. Histogram analysis of diffusion weighted imaging at $3 \mathrm{~T}$ is useful for prediction of lymphatic metastatic spread, proliferative activity, and cellularity in thyroid cancer. Int J Mol Sci. 2017, 18: 821.

24. Wang F, Wang Y X, Zhou Y, et al. Apparent diffusion coefficient histogram analysis for assessing tumor staging and detection of lymph node metastasis in epithelial ovarian cancer: correlation with p53 and Ki-67 expression. Mol Imaging Biol. 2019, 21: 731-739.

25. Moteki T, Horikoshi H, Endo K. Relationship between apparent diffusion coefficient and signal intensity in endometrial and other pelvic cysts. Magn Reson Imaging. 2002, 20: 463-470.

26. Yumiko O T, Satoshi O, Takako Y, et al. MRI of endometriotic cysts in association with ovarian carcinoma. AJR Am J Roentgenol. 2010, 194: 355-361.

27. Kato N, Sasou S, Motoyama T. Expression of hepatocyte nuclear factor-1beta (HNF-1beta) in clear cell tumors and endometriosis of the ovary. Mod Pathol. 2006, 19: 83-89.

28. Xiao W B, Awadallah A, Xin W. Loss of ARID1A/BAF250a expression in ovarian endometriosis and clear cell carcinoma. Int J Clin Exp Pathol. 2012, 5: 642-650.

\section{Figures}
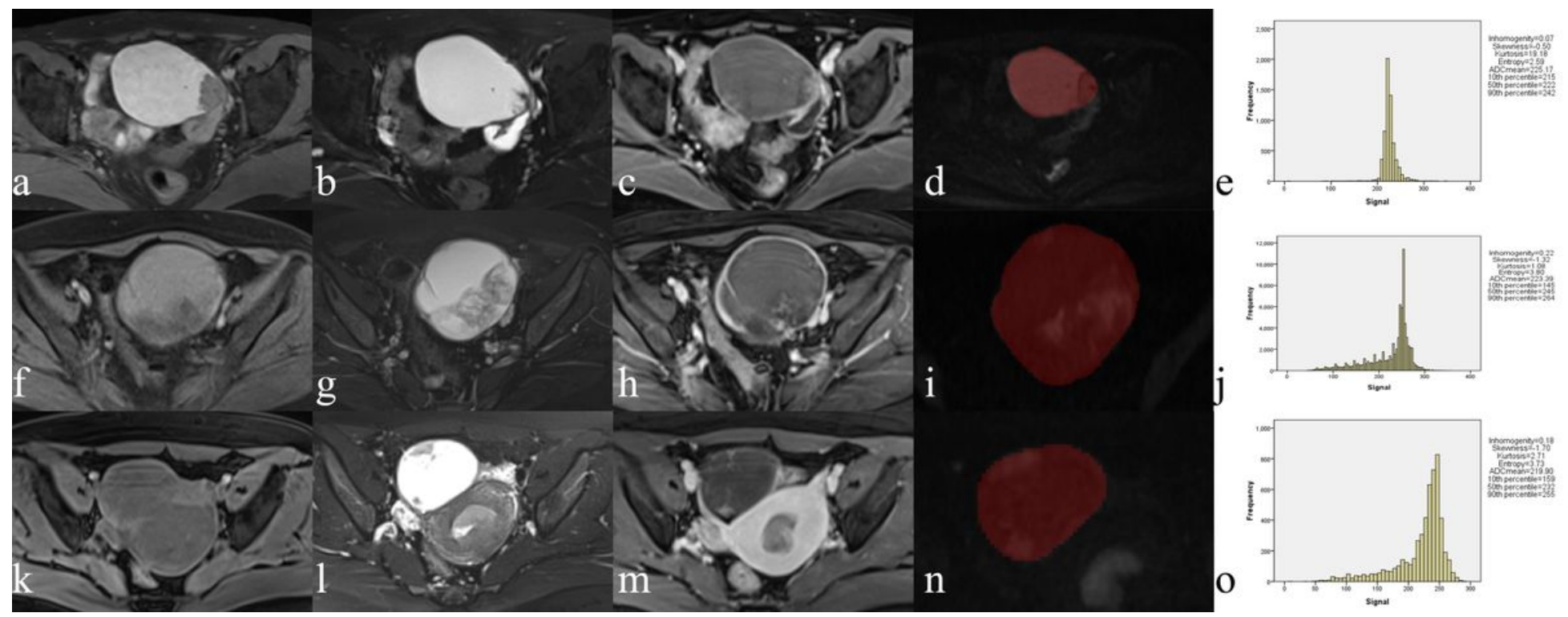

Page 9/10 


\section{Figure 1}

Whole-tumor ADC histogram analysis in patients with SMBT (a-e), CCC (f-j) and EC (k-o). Referring to T1WI (a, f, k), T2WI (b, g, I) and gadoliniumenhanced T1WI (c,h, m), the ROIs of the whole ovarian tumor were delineated slice-by-slice along the contour of the tumor manually on the ADC map $(b=1000 \mathrm{~s} / \mathrm{mm} 2)(\mathrm{d}, \mathrm{i}, \mathrm{n})$, and the histograms of gray-level distribution were calculated with the parameters listed in the figures (e, $\mathrm{j}$, o). The $x$-axis and $y$-axis in the histogram represent the voxel gray-level intensity and frequency of occurrence, respectively. ADC, apparent diffusion coefficient; SMBT, seromucinous borderline tumor; CCC, clear cell carcinoma; EC, endometrioid carcinoma; T1WI, T1-weighted imaging; T2WI, T2weighted imaging; ROI, region of interest.
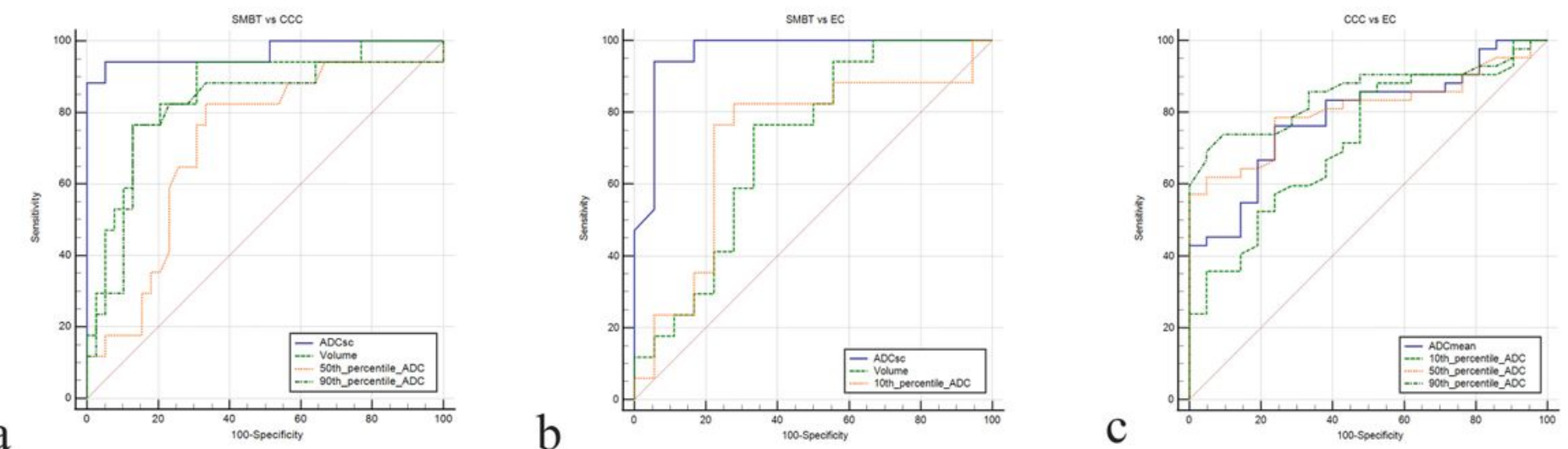

Figure 2

ROC curves of ADCSC and whole-tumor histogram-derived parameters for differentiation of SMBT, CCC and EC. a, ROC curve for differentiating between SMBT and CCC. The AUCs of ADCSC, volume, 50th and 90th percentile ADCs were 0.97, 0.86, 0.72 and 0.81, respectively, with no significant difference between AUCs of ADCSC and volume $(p=0.097)$ or the 90th percentile ADC $(p=0.053)$, and with significant difference between AUCs of ADC and the 50th percentile ADC ( $p=0.003)$. b, ROC curve for differentiating between SMBT and EC. The AUC of ADCSC was 0.97 , significantly higher than volume of $0.71(p=0.003)$ and the 10 th percentile ADC of $0.72(p=0.015)$. $c, R O C$ curve for differentiating between CCC and EC. The AUCs of the ADCmean, 10th, 50th and 90th percentile ADCs were $0.79,0.72,0.81$ and 0.85 , respectively, with no significant difference among them (all $p>0.05$ ). ADCSC, ADC of solid components; ROC, receiver operating characteristic; AUC, area under the curve. 